\title{
ON THE STABILITY OF DISCRETIZATIONS ${ }^{1}$
}

MARTIN H. SCHULTZ

Communicated by Fritz John, April 13, 1966

This paper is concerned with a study of some aspects of the stability question for finite difference methods for the integration of a system of linear ordinary differential equations with constant coefficients. Such a system may be written as follows:

$$
\begin{aligned}
d u(t) / d t & =M u(t), \quad t \geqq 0, \\
u(0) & =u_{0},
\end{aligned}
$$

where $M$ is a given $n \times n$ complex matrix, $u(t) \in R_{n}$ for all $t \geqq 0$, and $u_{0}$ is a given vector in $R_{n}$.

The discrete counterpart of the problem (1), (2) to be considered here is a one-parameter family of discretizations of the form

$$
\begin{aligned}
u_{k}(t+k) & =S(k) u_{k}(t), \quad t \geqq 0, \\
u_{k}(0) & =u_{0},
\end{aligned}
$$

where $k$ is a positive real parameter tending to $0, S(k)$ is an $n \times n$ complex matrix depending only on $k$, and $u_{k}(t) \in R_{n}$ for all $t \geqq 0, k>0$.

DEFINITION 1. The one-parameter family of discretizations (3), (4) is said to be consistent with (1), (2) if and only if

(5) $\|(S(k)-I) / k-M\| \rightarrow 0 \quad$ as $k \rightarrow 0 \quad$ for any matrix norm.

Various types of stability are introduced in

Definition 2. A one-parameter family of discretizations, $\{B(k)\}$, is said to be

(1) stable if and only if for any $T>0$ there exist positive constants $C$ and $k_{0}$ such that $\left\|S^{n}(k)\right\| \leqq C$ for all $0<k<k_{0}$ and all positive integers $n$ such that $n k \leqq T$,

(2) strictly stable if and only if there exists positive constants $k_{0}$ and $C$ such that $\left\|S^{n}(k)\right\| \leqq C$ for all $0<k<k_{0}$ and all positive integers $n$. If $k_{0}$ is the largest such constant, then $\left(0, k_{0}\right]$ is called the interval of strict stability.

(3) completely strictly stable if and only if there exists a positive constant $C(k)$ such that $\left\|S^{n}(k)\right\| \leqq C(k)$ for all $k>0$ and all positive integers $n$.

(4) uniformly strictly stable if and only if there exists a positive constant $C$ such that $\left\|S^{n}(k)\right\| \leqq C$ for all positive $k$ and nonnegative integers $n$.

1 This research was supported in part by NSF Grant GP-5553. 
The following result is obvious.

LEMMA 1. Uniform strict stability implies complete strict stability implies strict stability implies stability.

THEOREM 1. Every one-parameter family of discretizations, $\{S(k)\}$, consistent with (1), (2) is stable.

Proof. Condition (5) implies $\|S(k)\|=1+0(k)$ as $k \downarrow 0$, i.e., there exist positive constants $k_{1}, K$ such that $(\|\mathrm{S}(k)\|-1) / k<K$ for all $0<k<k_{1}$. Given $T>0$, let $n>0$ and $k>0$ be such that $n k \leq T$ and $k<k_{1}$. Then $\left\|S^{n}(k)\right\| \leqq\|S(k)\|^{n} \leqq e^{k t}$. QED.

The following classical result will be useful. For a proof see [3].

TheOREM 2. Let $M$ be an $n \times n$ complex matrix. Then,

(A) the set $\left\{M^{n}\right\}$ is equicontinuous with respect to $n \geqq 0$ if and only if $\rho(M)=\max _{1 \leqq i \leqq n}\left|\lambda_{i}(M)\right| \leqq 1$, where $\lambda_{i}(M)$ is an eigenvalue of $M$, and for every eigenvalue $\lambda_{j}$ with $\left|\lambda_{j}\right|=1$ and multiplicity $m_{j}$ there are $m_{j}$ corresponding eigenvectors,

(B) $\left\{e^{M t}\right\}$ is equicontinuous with respect to $t \geqq 0$, i.e., $M$ is the infinitesimal generator of an equicontinuous $C_{0}$-semigroup, if and only if $\sigma(M)=\max _{1 \leq i \leqq n} \operatorname{Re} \lambda_{i} \leqq 0$ and for every eigenvalue $\lambda_{j}$ with $\operatorname{Re}$ $\lambda_{j}=0$ and multiplicity $m_{j}$, there are $m_{j}$ corresponding eigenvectors.

In this paper $A(n)$ will denote the set of $n \times n$ complex matrices satisfying the conditions of Theorem $2(\mathrm{~A})$ and $B(n)$ the set of $n \times n$ complex matrices satisfying the conditions of Theorem $2(\mathrm{~B})$.

A well-known theorem of semigroup theory states that $M \in B(n)$ if and only if the one-parameter family of discretizations formed from the backwards difference approximation is uniformly strictly stable (cf. [2]). This result has led to the following two conjectures: If $M \in B(n)$, then

(I) every one-parameter family of discretizations consistent to (1), (2) is strictly stable,

(II) "implicit" one-parameter families of discretizations consistent to (1), (2) are uniformly strictly stable.

It will be shown that even if $M \in B(n)$, there exists a consistent one-parameter family of discretizations of every stability type. In the course of this, counter examples to conjectures I and II will be given. First, some notation is introduced.

Definition 3. The function $R(z)$, analytic in a neighborhood of 0 , is said to be a consistent approximation to $e^{z}$ if and only if $R(z)$ has a power series development about $z=0$ which agrees through at least linear terms with the power series development of $e^{z}$.

If $R(z)$ is such an approximation then $\{R(k M)\}$ forms a oneparameter family of discretizations consistent with (1), (2). In particular, the following classes of approximations are important: 
Definition 4. Let $D_{-}$and $D_{p}$ be the classes of meromorphic complex-valued functions of a complex variable defined by $D_{-}=\{R(z) \mid R(z)$ analytic in a neighborhood of the left half plane and $|R(z)| \leqq 1$ for all $z$ such that $\operatorname{Re} z \leqq 0\}$,

$D_{p}=\{R(z) \mid R(z)$ analytic in a neighborhood of the negative real axis and $|R(z)| \leqq 1$ for all real $z$ such that $z \leqq 0\}$.

REMARKs. (1) It follows from a Lemma of Birkhoff and Varga, (cf. [1]), that all the diagonal Padé approximations to $e^{z}$ belong to $D_{-}$, it also follows by inspection that the backwards difference approximation belongs to $D_{-}$.

(2) It follows from a Theorem of Varga, (cf. [6]), that a Padé approximation to $e^{z}$ is in $D_{p}$ if and only if it is a diagonal or subdiagonal approximation.

Theorem 3. Let $M \in B(n)$. Then, $\operatorname{Re} \lambda(M)=0$ implies $\lambda=0$ if and only if the one-parameter family of discretizations for the forward difference approximation $\{I+k M\}$ is strictly stable. The interval of strict stability is $(0, \tau]$ where $\tau=\min \left\{\left(-2 \operatorname{Re} \lambda_{i} /\left|\lambda_{i}\right|{ }^{2}\right) \lambda_{i} \neq 0\right\}$, or $+\infty$ if this set is empty.

Proof. If: If the set $\left\{\lambda_{i} \mid \lambda_{i} \neq 0\right\}$ is empty there is nothing to prove. Otherwise there exists a constant $c>0$ such that min $\lambda_{i \neq 0}\left(-2 \operatorname{Re} \lambda_{i}\right.$ $\left./\left|\lambda_{i}\right|^{2}\right)>k>0$ implies $\left\|(I+k M)^{n}\right\|<C$ for all $n \geqq 0$ and hence $\rho(I+k M) \leqq 1$.

Only if: If the set $\left\{\lambda_{i} \mid \lambda_{i} \neq 0\right\}$ is empty, $\rho(I+k M)=1$ for all $k>0$ and $\tau=+\infty$. Otherwise it is easy to check that $\rho(I+k M) \leqq 1$ for all $0<k \leqq \min \lambda_{i \neq 0}\left(-2 \operatorname{Re} \lambda_{i} /\left|\lambda_{i}\right|^{2}\right)$. Since $S(k)=\operatorname{Sup} n>0\left\|(I+k M)^{n}\right\|$ is a continuous function with respect to $k$, it has a maximum on the compact interval $\left[0, \min _{\lambda_{i \neq 0}}\left(-2 \operatorname{Re} \lambda_{i} /\left|\lambda_{i}\right|^{2}\right)\right]$. QED.

Corollary 1. If $M \neq 0$, and $M \in B(n)$, then the one-parameter family of discretizations based on the forward difference method may be either strictly stable or not.

This shows that the first conjecture is false. The following counterexample shows that the second conjecture is also false.

CoUnTER-EXAMPLE. The one-parameter family of discretizations of (1), (2) with

$$
M=\left[\begin{array}{rr}
-1 & 1 \\
0 & -1
\end{array}\right] \in B(2)
$$

based on the Crank-Nicholson method is not uniformly strictly stable.

Proof. For the Crank-Nicholson method

$$
S(k)=\left[\begin{array}{cr}
1+\alpha & -\alpha \\
0 & 1+\alpha
\end{array}\right]^{-1}\left[\begin{array}{cc}
1-\alpha & \alpha \\
0 & 1-\alpha
\end{array}\right], \quad \alpha=k / 2 .
$$


The proof is completed by setting $k=n^{1 / 2}$ and showing that $\left\|S^{n}\left(n^{1 / 2}\right)\right\| \rightarrow \infty$ as $n \rightarrow \infty$. QED.

The most general statement one can hope to make is the following:

THEOREM 4. If $R(z)$ is a consistent approximation to $e^{z}$, then $\{R(k M)\}$ is completely strictly stable for every $M \in B(n)$ if and only if $R(z) \in D_{-}$.

Proof. If: The eigenvalues of $R(k M)$ are of the form $R(k \lambda)$ where $\lambda$ is an eigenvalue of $M$. By Theorem $2(\mathrm{~B}), \operatorname{Re} \lambda \leqq 0$ with equality only if the number of eigenvectors corresponding to $\lambda$ equals the multiplicity of $\lambda$. Since $R$ is in $D_{-}|R(k \lambda)|<1$ except when $\lambda=0$, then $|R(0)|=1$ with the number of eigenvectors corresponding to $R(0)$ equal to the multiplicity of $R(0)$. The result then follows by applying Theorem 2(A). QED.

Only if: The proof is by contradiction. If $R(z)$ is not in $D_{-}$, then one can construct a diagonal matrix, satisfying all the appropriate conditions, with an eigenvalue $\lambda$ such that $|R(k \lambda)|>1$ for some $k>0$. This is a contradiction. QED.

If $M$ should have the property that $\|M\|=\rho(M)$ for some matrix norm, e.g. if $M$ is Hermitian, then the following result holds:

Corollary 2. If $R(z) \in D_{-}$and $M \in B(n)$ is such that $\|M\|=\rho(M)$ for some matrix norm then $\{R(k M)\}$ is uniformly strictly stable.

The results may be strengthened when $M$ is Hermitian.

THEOREM 5. If $R(z)$ is a consistent approximation to $e^{z}$, then $\{R(k M)\}$ is uniformly strictly stable for every Hermitian $M \in B(n)$ if and only if $R(z) \in D_{p}$.

CoRollary 3. If $R(z)$ is a diagonal or subdiagonal Padé approximation to $e^{z}$, then $\{R(k M)\}$ is uniformly strictly stable for every Hermitian $M \in B(n)$.

\section{REFERENCES}

1. G. Birkhoff and R. Varga, Discretization errors for well-set Cauchy problems. I, J. Math. and Phys. 44 (1965), 1-23.

2. E. Hille and R. S. Phillips, Functional analysis and semi-groups, Amer. Math. Soc., Providence, R. I., 1957.

3. R. Oldenberger, Infinite powers of matrices and characteristic roots, Duke Math. J. 6 (1940), 357-361.

4. R. D. Richtmyer, Difference methods for initial value problems, Interscience, New York, 1957. 1962.

5. R. S. Varga, Matrix iterative analysis, Prentice-Hall, Englewood Cliffs, N. J.,

6. - - On higher order stable implicit methods for solving parabolic partial differential equations, J. Math. and Phys. 40 (1961), 220-231.

Case Institute of Technology 